ARTIGO ORIGINAL

\title{
Dinâmica de populações arbóreas em fitofisionomias de Cerrado e de Floresta Estacional Semidecidual
}

\author{
Dynamics of tree populations in Cerrado and seasonal semi-deciduous \\ forest phyto-physiognomies \\ Leovandes Soares da Silva' ${ }^{1}$, Thiago José Ornelas Otoni ${ }^{1}$ (), Arthur Duarte Vieira ${ }^{1}$ (D), \\ Anne Priscila Dias Gonzaga ${ }^{1}$ (D), Israel Marinho Pereira ${ }^{1}$ (D), Evandro Luiz Mendonça Machado ${ }^{1}$ (D), \\ Stênio Abdanur Porfírio Franco ${ }^{1}$ (1) \\ ${ }^{1}$ Programa de Pós-graduação em Ciência Florestal, Universidade Federal dos Valores do Jequitinhonha e Mucuri - UFVJM, \\ Diamantina, MG, Brasil
}

\begin{abstract}
Como citar: Silva, L. S., Otoni, T. J. O., Vieira, A. D., Gonzaga, A. P. D., Pereira, I. M., Machado, E. L. M., \& Franco, S. A. P. (2020). Dinâmica de populações arbóreas em fitofisionomias de Cerrado e de Floresta Estacional Semidecidual. Scientia Forestalis, 48(128), e3300. https://doi.org/10.18671/scifor.v48n128.23
\end{abstract}

\section{Resumo}

O objetivo desse estudo foi investigar, como os padrões temporais das espécies mais abundantes das comunidades determinam os processos de sucessão em diferentes formações vegetacionais do Bioma Cerrado. No primeiro inventário, em 2010 no Cerrado stricto sensu e no Cerradão, foram identificados e mensurados o diâmetro de todos os indivíduos a $30 \mathrm{~cm}$ acima do solo (DAS, 0,30m) $\geq 5,0 \mathrm{~cm}$. Já para a Floresta Estacional Semidecidual, em 2011, foram medidos e identificados todos os indivíduos vivos com diâmetro a altura do peito (DAP) $\geq 5,0 \mathrm{~cm}$. O segundo inventário nas três fitofisionomias foi realizado em 2015, adotando-se os mesmos critérios do inventário anterior. A mortalidade foi superior ao recrutamento, entre todas as populações estudadas, 15 populações não tiveram indivíduos recrutados e apenas duas espécies (Bowdichia virgilioides Kunth e Caryocar brasiliense Cambess) não perderam indivíduos. As mudanças nos parâmetros de dinâmica variaram entre as populações e o recrutamento foi maior nas populações com maior perda de indivíduos, isso indica que essas espécies, independente da fitofisionomia, possuem maior potencial de regeneração, pós-distúrbios.
\end{abstract}

Palavras-chave: Área basal; Guildas; Mudanças estruturais; Sucessão ecológica.

\begin{abstract}
The aim of this study was to investigate how the temporal patterns of the most abundant species in the communities determine the succession processes in different vegetation formations of the Cerrado Biome. In the first inventory, in 2010 in the Cerrado stricto sensu and Cerradão, the diameter of all individuals $30 \mathrm{~cm}$ above the ground (DAS, $0.30 \mathrm{~m}) \geq 5.0 \mathrm{~cm}$ was identified and measured. For the SSF in 2011, all living individuals with breast height diameter $(\mathrm{DBH}) \geq 5,0 \mathrm{~cm}$ were measured and identified. The second inventory in the three phyto-physiognomies was carried out in 2015, when the same criteria of the previous inventory were adopted. Mortality was higher than recruitment, in all populations studied, 15 populations had no individuals recruited and only two species (Bowdichia virgilioides Kunth and Caryocar brasiliense Cambess) did not lose individuals. Changes in dynamic parameters varied among populations and recruitment was higher in populations with the highest loss of individuals, indicating that these species, regardless of their phyto-physiognomy, have a greater regeneration potential after disturbances.
\end{abstract}

Keywords: Basal area; Guilds; Structural changes; Ecological succession.

Fonte de financiamento: Nenhuma.

Conflito de interesse: Nada a declarar.

Autor correspondente: leosoares.ef@gmail.com

Recebido: 26 fevereiro 2019.

Aceito: 26 novembro 2019.

Editor: Paulo Henrique Müller Silva.

(i) Este é um artigo publicado em acesso aberto (Open Access) sob a licença Creative Commons Attribution, que permite uso, distribuição e reprodução cc. em qualquer meio, sem restrições desde que o trabalho original seja corretamente citado. 


\section{INTRODUÇÃO}

O processo de estabelecimento das diferentes espécies e consequentemente das diversas fitofisionomias parece ser produto da combinação de fatores edáficos, climáticos e geográficos, como também dos efeitos antrópicos que alteram a paisagem e a permeabilidade de espécies nas comunidades florestais (Enoki et al., 2014; Silva et al., 2017). Ehrlén \& Morris (2015) atribuem as mudanças que ocorrem na estrutura e no arranjo das populações vegetais aos resultados de ações de forças bióticas e abióticas, como também na interação destes entre si e com a paisagem.

As populações vegetais passam por mudanças contínuas no tempo em função das taxas de nascimento, crescimento e morte dos indivíduos (Mews et al., 2011). Tais mudanças podem ser medidas e descritas pelas variáveis biométricas que imprimem a estrutura da comunidade em um dado momento, podendo-se entender sua evolução por meio das avaliações de dinâmica da vegetação (Paiva et al., 2007; Mews et al., 2011). Estudos dessa natureza permitem também, o entendimento da capacidade de resiliência de comunidades pósdistúrbios ocasionais (Melo \& Durigan, 2010; Cubas et al., 2016).

As comunidades vegetais não são entidades estacionárias e suas sucessões são intrínsecas, bem como decorridas de fatores sinérgicos do meio abiótico, além da presença de distúrbios naturais ou não (Appollinário et al., 2005). Assim, o sentido das mudanças e suas velocidades dependem da intensidade e tempo de duração das alterações ambientais e da resposta do meio biótico aos processos de reprodução pós-distúrbio, como por exemplo, a brotação de troncos e raízes, banco de sementes no solo, e da entrada de sementes e propágulos (Appollinário et al., 2005).

Os incêndios, causas frequentes de alterações ambientais em comunidades naturais, podem provocar diversas mudanças na composição e estrutura da vegetação, que estão relacionadas à intensidade e à duração deste evento (Lima et al., 2009). Além do fogo, outros fatores podem contribuir para essas mudanças, e por essa razão, estudos em escala temporal são essenciais para o melhor entendimento das variações vegetacionais locais (Rios et al., 2018).

Estudos de dinâmica florestal, incluso pós-distúrbio, podem elucidar as estratégias de sobrevida selecionadas pelas populações vegetais, subsidiando previsões da transformação estrutural e da composição de comunidades (Higuchi et al., 2008). Neste sentido, o presente trabalho investigou como os padrões temporais das espécies mais abundantes das comunidades determinam os processos de sucessão em diferentes formações vegetacionais do Bioma Cerrado.

\section{MATERIAL E MÉTODOS}

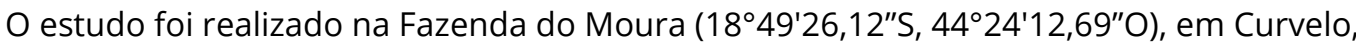
Minas Gerais. A área de estudo está inserida na bacia do Rio São Francisco, em altitude de $715 \mathrm{~m}$ e aproximadamente 436 ha. Segundo a classificação de Köppen o clima na região é do tipo Aw - tropical com inverno seco, com índice pluviométrico próximo a $1200 \mathrm{~mm}$ anuais e temperatura média anual em torno de $28^{\circ} \mathrm{C}$ (Alvares et al., 2013).

$\mathrm{Na}$ área de estudo ocorre três distintas fitofisionomias: cerrado stricto sensu - CSS, cerradão - CD, segundo a proposta de Ribeiro \& Walter (2008) e Floresta Estacional Semidecidual - FES, segundo Veloso et al. (1991).

O CSS $\left(18^{\circ} 50^{\prime} 7,26^{\prime \prime} S, 44^{\circ} 23^{\prime} 33,49^{\prime \prime}\right.$ ) apresenta substrato distrófico e Latossolo ácido, ocupando cerca de 60 ha. O CD $\left(18^{\circ} 49^{\prime} 56,11^{\prime \prime} \mathrm{S}, 44^{\circ} 23^{\prime} 6,42^{\prime \prime} \mathrm{O}\right)$ apresenta área de aproximadamente 220 ha e o solo foi caracterizado, de forma geral, como Latossolo vermelho distrófico. A FES (18 $\left.49^{\prime} 21,17^{\prime \prime} \mathrm{S}, 44^{\circ} 23^{\prime} 55,9^{\prime \prime} \mathrm{O}\right)$ possui aproximadamente 162 ha e se encontra margeada pelas fitofisionomias CSS e CD, além de áreas modificadas pela agricultura. Esta última possui em seu interior curso d'água formando gradiente de umidade em direção à borda. Nessa fitofisionomia ocorreu queda de indivíduos em uma parcela, decorrente da ação do vento após o primeiro inventário. 
Cabe a ressalva que a fazenda foi atingida por um incêndio, logo após o primeiro inventário, realizado em 2010. Durante esse evento, nas fitofisionomias CSS e CD o fogo se propagou ao longo de boa parte das suas áreas, sendo este visualmente mais intenso no CD, em razão da maior quantidade de indivíduos mortos ali encontrados. A FES foi a comunidade menos afetada, sendo observado vestígios da passagem do fogo somente nas bordas.

\section{Amostragem e coleta dos dados}

Tanto o CSS quanto o CD tiveram o primeiro inventário realizado por Otoni et al. (2013). $\mathrm{Na}$ ocasião, foram alocadas 15 e 10 parcelas permanentes de $20 \times 50 \mathrm{~m}$, respectivamente no CSS e CD, e estas foram distribuídas de forma sistemática e distanciadas $100 \mathrm{~m}$ entre si. Em cada parcela foram identificados e mensurados todos os indivíduos arbustivo-arbóreos vivos com diâmetro a $30 \mathrm{~cm}$ acima do nível do solo (DAS) $\geq 5,0 \mathrm{~cm}$. Os indivíduos com troncos múltiplos foram mensurados quando a raiz da soma dos quadrados dos DAS foi $\geq 5,0 \mathrm{~cm}$ (diâmetro quadrático).

A FES foi inventariada em 2011, em 25 parcelas de $10 \times 40$ m, separadas entre si por $30 \mathrm{~m}$. Nas parcelas foram mensurados e identificados todos os indivíduos arbustivo-arbóreos vivos com diâmetro a altura do peito (DAP) $\geq 5,0 \mathrm{~cm}$.

Nas três fitofisionomias as espécies foram identificadas por meio de consultas à literatura e especialistas, ou por comparações com exsicatas existentes no Herbário Dendrológico Jeanine Felfili (HDJF) da Universidade Federal dos Vales do Jequitinhonha e Mucuri (UFVJM). As espécies foram agrupadas em famílias seguindo o sistema APG IV (Angiosperm Phylogeny Group, 2016). A verificação da grafia e atualização dos nomes científicos das espécies foram feitas com base nos dados da Flora do Brasil 2020.

Em 2015 foi realizado o segundo inventário, quando foram avaliados novamente todos os indivíduos das parcelas das três fitofisionomias. Assim sendo, todos os indivíduos vivos (sobreviventes) foram remedidos e os mortos foram registrados. Aqueles que atingiram o critério de inclusão neste levantamento (recrutas) foram mensurados e receberam placas numeradas para acompanhamento ao longo do tempo.

\section{Dinâmica das populações arbustivo-arbóreas}

A dinâmica das três fitofisionomias foi analisada para as populações mais abundantes, sendo estas formadas pelas espécies que apresentaram vinte ou mais indivíduos nos dois inventários, incluindo mortos e recrutas.

A análise da dinâmica foi feita segundo modelos algébricos propostos por Sheil \& May (1996) e Sheil et al. (2000). Desta forma, foram calculadas as taxas anuais médias de mortalidade (M) e recrutamento (R) das espécies, assim como as taxas anuais médias de perda $(P)$ e ganho $(G)$ de área basal das árvores, por meio de expressões exponenciais.

$$
\begin{aligned}
& M=\left(1-\left[\left(\mathrm{N}_{0}-\mathrm{m}\right) / \mathrm{N}_{0}\right)^{1 / t}\right] \times 100 \\
& \mathrm{R}=\left[1-\left(1-\mathrm{r} / \mathrm{N}_{\mathrm{f}}\right)^{1 / \mathrm{t}}\right] \times 100 \\
& \mathrm{P}=\left\{1-\left[\left(\mathrm{AB}_{0}-\mathrm{AB}_{\mathrm{m}} \mathrm{AB}_{\mathrm{d}}\right) / \mathrm{AB}_{0}\right]^{1 / t}\right\} \times 100 \\
& \mathrm{G}=\left\{1-\left[1-\left(\mathrm{AB}_{\mathrm{r}}+\mathrm{AB}_{\mathrm{g}}\right) / \mathrm{AB}_{\mathrm{f}}\right]^{1 / \mathrm{t}}\right\} \times 100
\end{aligned}
$$

Em que: $N_{0}$ e $N_{f}=$ número de indivíduos nos inventários inicial e final respectivamente, $t=$ tempo entre os inventários inicial e final, $A_{0}$ e $A B_{f}=$ áreas basais dos indivíduos nos 
inventários inicial e final, respectivamente, $A_{m}$ e $A B_{r}=$ área basal dos mortos e ingressantes, respectivamente, $A B_{d}-A B_{g}=$ área basal do decremento e do incremento.

Foram também calculadas as taxas de mudança líquida no período, realizadas conforme Korning \& Balslev (1994), para área basal $\left(\mathrm{Ch}_{\mathrm{AB}}\right)$ e para número de árvores $\left(\mathrm{Ch}_{\mathrm{N}}\right)$. Para verificar a diferença entre o número de recrutas e mortos foram utilizadas contagens de Poisson (Zar, 1996), por populações.

$$
\begin{aligned}
& \mathrm{Ch}_{\mathrm{AB}}=\left[\left(\mathrm{AB}_{\mathrm{f}} / \mathrm{AB}_{0}\right)^{1 / \mathrm{t}}-1\right] \times 100 \\
& \mathrm{Ch}_{\mathrm{N}}=\left[\left(\mathrm{N}_{\mathrm{f}} / \mathrm{N}_{0}\right)^{1 / \mathrm{t}}-1\right] \times 100
\end{aligned}
$$

Em que: $\mathrm{t}=$ tempo entre os inventários inicial e final, $\mathrm{N}_{0}$ e $\mathrm{N}_{\mathrm{f}}=$ número de indivíduos nos inventários inicial e final, respectivamente, $A_{0}$ e $A B_{f}=$ áreas basais dos indivíduos no primeiro e no segundo inventário.

Visando melhor descrever as mudanças nas comunidades ao longo do intervalo de tempo avaliado, as espécies das populações selecionadas foram classificadas quanto ao seu estágio sucessional e guildas de dispersão. A classificação sucessional foi realizada conforme os grupos ecológicos sugeridos por Gandolfi et al. (1995), sendo portanto, as espécies diferenciadas em: pioneiras (P) - espécies que se desenvolvem em clareiras, nas bordas da floresta ou em locais abertos, sendo claramente dependentes de condições de maior luminosidade, secundárias iniciais (SI) - espécies que se desenvolvem em clareiras pequenas ou mais raramente no sub-bosque, em condições de algum sombreamento, secundárias tardias (ST) - espécies que se desenvolvem exclusivamente em sub-bosque permanentemente sombreado e, nesse caso, pequenas árvores ou espécies arbóreas de grande porte que se desenvolvem lentamente em ambientes sombreados, e (SC) - sem caracterização. Estas classificações foram feitas com base em consultas bibliográficas.

Para classificação das espécies em guildas de dispersão, foram utilizadas as categorias e critérios de Van der Pijl (1982) para definir as seguintes síndromes: (a) anemocóricas - espécies cujos diásporos são disseminados pelo vento, (b) autocóricas - as que dispersam suas sementes pela gravidade ou por deiscência explosiva, e (c) zoocóricas - aquelas cujos diásporos são disseminados por animais. Para esta classificação, foram utilizadas informações sobre a biologia das espécies na literatura (Lopes et al., 2011; Miguel et al., 2016; Nunes et al., 2003; Oliveira-Filho, 2006; Pereira et al., 2010; Toniato \& Oliveira-Filho, 2004) e acrescidas do conhecimento dos próprios autores.

\section{RESULTADOS E DISCUSSÃO}

Foram amostradas 28 espécies no CSS, seguido de CD (16) e FES (11). Dez populações foram encontradas concomitantemente no CSS e CD e apenas Protium heptaphyllum esteve entre as populações selecionadas simultaneamente no CD e na FES (Tabela 1). Nenhuma população selecionada esteve presente nas três fitofisionomias ao mesmo tempo.

Tabela 1. Dinâmica de populações arbóreas inventariadas na Fazenda do Moura, Curvelo (MG). Fito. =

\begin{tabular}{|c|c|c|c|c|c|c|c|c|c|c|c|}
\hline \multirow{2}{*}{ Espécies } & \multirow{2}{*}{ Fito. } & \multicolumn{6}{|c|}{ Número de árvores } & \multirow{2}{*}{ TMNi } & \multicolumn{2}{|c|}{ Área basal $\left(\mathrm{m}^{2}\right)$} & \multirow{2}{*}{ TMAB } \\
\hline & & N1 & N2 & $\mathbf{M}$ & $\mathbf{R}$ & $\mathbf{Z}$ & $\boldsymbol{P}$ & & AB 1 & AB 2 & \\
\hline Annona crassiflora Mart. & CSS & 28 & 28 & 2 & 2 & 0,00 & ns & 0,00 & 0,577 & 0,557 & $-0,69$ \\
\hline Annona sylvatica A. St.-Hil. & FES & 24 & 21 & 3 & 0 & 1,73 & 0,10 & $-2,64$ & 0,309 & 0,320 & 0,73 \\
\hline Aspidosperma tomentosum Mart & CSS & 77 & 79 & 6 & 8 & 0,53 & ns & 0,51 & 0,245 & 0,267 & 1,69 \\
\hline \multirow{2}{*}{ Astronium fraxinifolium Schott } & $C D$ & 46 & 34 & 13 & 1 & 3,21 & 0,002 & $-5,87$ & 0,380 & 0,362 & $-0,96$ \\
\hline & CSS & 99 & 95 & 7 & 3 & 1,26 & ns & $-0,82$ & 0,657 & 0,700 & 1,27 \\
\hline Bowdichia virgilioides Kunth & CSS & 37 & 37 & 0 & 0 & -- & -- & 0,00 & 0,625 & 0,793 & 4,89 \\
\hline
\end{tabular}
Fitofisionomias, CSS = Cerrado stricto sensu, CD = Cerradão, FES = Floresta Estacional Semidecidual. 
Tabela 1. Continuação...

\begin{tabular}{|c|c|c|c|c|c|c|c|c|c|c|c|}
\hline \multirow{2}{*}{ Espécies } & \multirow{2}{*}{ Fito. } & \multicolumn{6}{|c|}{ Número de árvores } & \multirow{2}{*}{ TMNi } & \multicolumn{2}{|c|}{ Área basal $\left(\mathrm{m}^{2}\right)$} & \multirow{2}{*}{ TMAB } \\
\hline & & N1 & N2 & M & $\mathbf{R}$ & z & $\boldsymbol{P}$ & & AB 1 & AB 2 & \\
\hline Byrsonima coccolobifolia Kunth & CSS & 110 & 101 & 9 & 0 & 3,00 & 0,005 & $-1,69$ & 0,822 & 0,882 & 1,42 \\
\hline Byrsonima verbascifolia (L.) DC. & CSS & 43 & 40 & 3 & 0 & 1,73 & 0,10 & $-1,44$ & 0,303 & 0,344 & 2,57 \\
\hline Caryocar brasiliense Cambess. & CSS & 35 & 37 & 0 & 2 & 1,41 & ns & 1,12 & 2,693 & 2,846 & 1,11 \\
\hline Copaifera langsdorffii Desf. & FES & 34 & 30 & 4 & 0 & 2,00 & 0,05 & $-2,47$ & 4,309 & 4,373 & 0,29 \\
\hline Coutarea hexandra (Jacq.) K. Schum. & FES & 38 & 36 & 2 & 0 & 1,41 & ns & $-1,08$ & 0,242 & 0,254 & 1,00 \\
\hline Diospyros burchellii Hiern & CSS & 51 & 43 & 13 & 5 & 1,89 & 0,10 & $-3,35$ & 0,389 & 0,412 & 1,13 \\
\hline Erythroxylum deciduum A. St.-Hil. & CSS & 56 & 56 & 11 & 11 & 0,00 & ns & 0,00 & 0,242 & 0,256 & 1,17 \\
\hline Erythroxylum suberosum A. St.-Hil. & CSS & 141 & 122 & 24 & 5 & 3,53 & 0,001 & $-2,85$ & 0,534 & 0,536 & 0,07 \\
\hline Eugenia acutata Miq. & FES & 80 & 81 & 4 & 5 & 0,33 & ns & 0,25 & 0,748 & 0,795 & 1,23 \\
\hline Faramea hyacinthina Mart. & FES & 54 & 57 & 2 & 5 & 1,13 & ns & 1,09 & 0,716 & 0,784 & 1,83 \\
\hline $\begin{array}{c}\text { Hyptidendron canum (Pohl ex } \\
\text { Benth.) Harley }\end{array}$ & $C D$ & 47 & 20 & 28 & 1 & 5,01 & 0,001 & $-15,7$ & 0,217 & 0,134 & $-9,17$ \\
\hline Kielmeyera coriacea Mart. \& Zucc. & CSS & 224 & 200 & 27 & 3 & 4,38 & 0,001 & $-2,24$ & 1,354 & 1,386 & 0,47 \\
\hline $\begin{array}{l}\text { Kielmeyera grandiflora (Wawra) } \\
\text { Saddi }\end{array}$ & $C D$ & 88 & 55 & 33 & 0 & 5,74 & 0,001 & $-8,97$ & 0,517 & 0,426 & $-3,81$ \\
\hline $\begin{array}{l}\text { Lafoensia vandelliana Cham. \& } \\
\text { Schltdl }\end{array}$ & CSS & 57 & 42 & 15 & 0 & 3,87 & 0,001 & $-5,92$ & 0,313 & 0,251 & $-4,29$ \\
\hline \multirow{2}{*}{ Leptolobium dasycarpum Vogel } & CSS & 24 & 23 & 1 & 0 & 1,00 & ns & $-0,85$ & 0,250 & 0,293 & 3,18 \\
\hline & $C D$ & 71 & 32 & 40 & 1 & 6,09 & 0,001 & $-14,7$ & 0,480 & 0,274 & $-10,6$ \\
\hline \multirow{2}{*}{ Machaerium opacum Vogel } & $C D$ & 45 & 25 & 20 & 0 & 4,47 & 0,001 & $-11,0$ & 0,338 & 0,295 & $-2,70$ \\
\hline & CSS & 48 & 48 & 4 & 4 & 0,00 & ns & 0,00 & 0,442 & 0,592 & 6,03 \\
\hline \multirow[t]{2}{*}{ Magonia pubescens A. St.-Hil. } & $C D$ & 472 & 354 & $\begin{array}{c}12 \\
6\end{array}$ & 8 & 10,1 & 0,001 & $-5,59$ & 2,957 & 2,436 & $-3,80$ \\
\hline & CSS & 833 & 815 & 49 & 31 & 2,01 & 0,05 & $-0,44$ & 3,508 & 3,674 & 0,93 \\
\hline Maytenus gonoclada Mart. & FES & 37 & 34 & 4 & 1 & 1,34 & ns & $-1,68$ & 0,522 & 0,543 & 0,79 \\
\hline Ocotea odorifera Rohwer & FES & 27 & 25 & 3 & 1 & 1,00 & ns & $-1,53$ & 0,332 & 0,302 & $-1,85$ \\
\hline Ouratea hexasperma (A. St.-Hil.) Baill. & CSS & 58 & 64 & 4 & 10 & 1,60 & ns & 1,99 & 0,263 & 0,315 & 3,70 \\
\hline Piptocarpha rotundifolia (Less.) Baker & CSS & 62 & 60 & 15 & 13 & 0,38 & ns & $-0,65$ & 0,463 & 0,412 & $-2,28$ \\
\hline Plathymenia reticulata Benth. & CSS & 20 & 23 & 2 & 5 & 1,13 & ns & 2,83 & 0,382 & 0,450 & 3,36 \\
\hline Plenckia populnea Reissek. & CSS & 46 & 46 & 3 & 3 & 0,00 & ns & 0,00 & 0,360 & 0,469 & 5,42 \\
\hline Pouteria ramiflora (Mart.) Radlk. & CSS & 97 & 93 & 5 & 1 & 1,63 & ns & $-0,84$ & 0,974 & 1,168 & 3,71 \\
\hline Protium heptaphyllum (Aubl.) & $C D$ & 86 & 77 & 19 & 10 & 1,67 & 0,10 & $-2,19$ & 0,621 & 0,714 & 2,83 \\
\hline Marchand & FES & 44 & 49 & 3 & 8 & 1,51 & ns & 2,18 & 1,208 & 1,226 & 0,30 \\
\hline Protium spruceanum (Benth.) Engl. & FES & 116 & 117 & 13 & 14 & 0,19 & ns & 0,17 & 2,490 & 2,569 & 0,62 \\
\hline Qualea multiflora Mart. & CSS & 49 & 41 & 11 & 3 & 2,14 & 0,05 & $-3,50$ & 0,290 & 0,271 & $-1,33$ \\
\hline \multirow{2}{*}{ Qualea grandiflora Mart. } & $C D$ & 211 & 167 & 46 & 2 & 6,35 & 0,001 & $-4,57$ & 3,375 & 3,161 & $-1,30$ \\
\hline & CSS & 129 & 126 & 14 & 11 & 0,60 & ns & $-0,47$ & 1,184 & 1,405 & 3,48 \\
\hline \multirow{2}{*}{ Qualea parviflora Mart. } & $C D$ & 84 & 58 & 27 & 1 & 4,91 & 0,001 & $-7,14$ & 0,808 & 0,678 & $-3,47$ \\
\hline & CSS & 283 & 273 & 21 & 11 & 1,77 & 0,10 & $-0,72$ & 2,603 & 2,906 & 2,23 \\
\hline Roupala montana Aubl. & $C D$ & 46 & 24 & 25 & 3 & 4,16 & 0,001 & $-12,2$ & 0,368 & 0,231 & $-8,92$ \\
\hline \multirow{2}{*}{ Tabebuia aurea (Silva Manso) } & $C D$ & 34 & 27 & 9 & 2 & 2,11 & 0,05 & $-4,51$ & 0,257 & 0,283 & 1,95 \\
\hline & CSS & 48 & 42 & 6 & 0 & 2,45 & 0,02 & $-2,64$ & 0,452 & 0,509 & 2,41 \\
\hline \multirow{2}{*}{ Tachigali aurea Tul. } & $C D$ & 46 & 39 & 10 & 3 & 1,94 & 0,10 & $-3,25$ & 0,448 & 2,394 & 39,81 \\
\hline & CSS & 34 & 32 & 2 & 0 & 1,41 & ns & $-1,21$ & 0,414 & 0,545 & 5,64 \\
\hline Tachigali vulgarisL.G.Silva \& H.C.Lima & $C D$ & 206 & 122 & 87 & 3 & 8,85 & 0,001 & $-9,95$ & 2,834 & 4,293 & 8,66 \\
\hline \multirow{2}{*}{ Terminalia argentea Mart. } & $C D$ & 77 & 65 & 14 & 2 & 3,00 & 0,005 & $-3,33$ & 1,627 & 1,607 & $-0,25$ \\
\hline & CSS & 37 & 36 & 1 & 0 & 1,00 & ns & $-0,55$ & 0,385 & 0,426 & 2,08 \\
\hline Terminalia glabrescens Mart. & FES & 29 & 23 & 6 & 0 & 2,45 & 0,02 & $-4,53$ & 1,363 & 1,164 & $-3,11$ \\
\hline $\begin{array}{c}\text { Tocoyena formosa (Cham. \& Schltdl.) } \\
\text { K. Schum. }\end{array}$ & CSS & 22 & 22 & 2 & 2 & 0,00 & ns & 0,00 & 0,085 & 0,096 & 2,57 \\
\hline Trichilia pallens C. DC. & FES & 35 & 32 & 3 & 0 & 1,73 & 0,10 & $-1,78$ & 0,489 & 0,544 & 2,18 \\
\hline \multirow{2}{*}{ Vatairea macrocarpa (Benth.) Ducke } & $C D$ & 36 & 27 & 12 & 3 & 2,32 & 0,05 & $-5,59$ & 0,463 & 0,505 & 1,73 \\
\hline & CSS & 91 & 84 & 8 & 1 & 2,33 & 0,02 & $-1,59$ & 0,705 & 0,822 & 3,14 \\
\hline Xylopia aromática (Lam.) Mart. & $C D$ & 65 & 27 & 40 & 2 & 5,86 & 0,001 & $-16,1$ & 0,401 & 0,315 & $-4,74$ \\
\hline
\end{tabular}

$\mathrm{N} 1$ = inventário inicial, $\mathrm{N} 2$ = inventário final, $\mathrm{M}=$ mortos, $\mathrm{R}=$ recrutas. Comparações de contagens de Poisson entre $\mathrm{M}$ e R: valores de $Z$ e sua significância $(p)$. TMNi = taxa de mudanças em número de indivíduos. AB1 = inventário inicial, $\mathrm{AB} 2$ = inventário final, $\mathrm{TMAB}=$ taxa de mudança em área basal. 
Analisando por fitofisionomia, por meio da contagem de Poisson, das 28 populações do CSS, em 16 populações $(57,1 \%)$ as mudanças entre mortalidade e recrutamento foram positivas e não significativas, porém em 11 delas (39,2\%) foram positivas, porém significativas. O recrutamento foi superior a mortalidade nas populações de Plathymenia reticulata, Ouratea hexasperma, Caryocar brasiliense e Aspidosperma tomentosum, e nessas populações houve ganho em área basal. Em 15 populações não houve recrutamento e duas espécies não perderam indivíduos, a saber, Bowdichia virgilioides e Caryocar brasiliense, tendo esta última ganhando dois indivíduos. Para a população de Bowdichia virgilioides não foram detectadas mudanças entre a mortalidade e o recrutamento, porém, houve aumento em área basal (Tabela 1).

Para o número de indivíduos foi observado que 18 populações apresentaram taxas de mudanças negativas. Enquanto que outras quatro populações tiveram taxas positivas e em seis delas não foram detectadas mudanças. Já para a área basal, 24 populações tiveram as taxas de mudanças positivas, sendo observadas nas quatro restantes mudanças negativas. Aqui cabe ressaltar que o acréscimo em área basal, dentre as fitofisionomias, foi mais pronunciado nas populações do CSS (Tabela 1).

No CD, pela contagem de Poisson, as mudanças entre mortalidade e recrutamento foram significativas em todas as populações, sendo a mortalidade superior ao recrutamento em todas elas, assim sendo, para esta fitofisionomia foi registrada mudança negativa. Cinco populações no CD apresentaram mudanças positivas para a área basal, destaque para a população de Tachigali aurea, que mesmo perdendo indivíduos foi a população com maior incremento em área basal (Tabela 1).

As populações de Xylopia aromatica, Roupala montana, Hyptidendron canum e Leptolobium dasycarpum, além do baixo recrutamento, foram as que mais perderam em área basal, devido as altas taxas de mortalidade (Tabela 1). Já para a taxa de mudança em área basal, em 11 populações $(68,7 \%)$ estas foram negativas, ou seja, houve perda em biomassa. Enquanto que em outras cinco (31,3\%) foram registradas incremento em área basal (mudança positiva). Cabe destacar que Hyptidendron canum foi a população com as maiores perdas, tanto em indivíduos quanto em área basal (Tabela 1).

De maneira geral, o que se pode observar com a dinâmica populacional destas duas fitofisionomias foi a redução populacional entre os inventários. Este provavelmente foi decorrente dos distúrbios ocorridos após o primeiro inventário, principalmente o fogo (em CSS e CD). De fato, Gomes et al. (2014) e Lenza et al. (2017), afirmaram que os incêndios diminuem a densidade em fitofisionomias do Cerrado. Além disso, em estudos realizados em áreas de Cerrado stricto sensu no Distrito Federal, o fogo foi o principal responsável pela redução da vegetação lenhosa (Felfili et al., 2000).

Assim, acredita-se que os espaços criados pela morte dos indivíduos, a ciclagem de nutrientes acelerada pelo fogo, a redução na densidade de indivíduos de outras espécies e consequente redução da competição tenham contribuído para o incremento dos sobreviventes. Sendo as populações de Tachigali vulgaris e Tachigali aurea no CD as que mais aproveitaram essas condições, registrando acentuado crescimento diamétrico (Tabela 1).

Esses resultados corroboram com o trabalho apresentado por Lima et al. (2009), que afirmaram que a abertura da vegetação causada pelo fogo contribui para o incremento em área basal dos indivíduos sobreviventes. Isso porque, a disponibilidade de nutrientes no solo pode variar, segundo a frequência das queimadas, com a disponibilidade de nutrientes tendendo a aumentar conforme aumenta a frequência das queimadas (Pivello et al., 2010). Nesse caso, as espécies mais resistentes ao fogo seriam as mais beneficiadas com aumento dos nutrientes no solo.

$\mathrm{Na}$ maioria das populações aqui investigadas, as taxas de recrutamento foram insuficientes para compensar a mortalidade. Esse fato pode estar relacionado a processos de reprodução, estresses e, ou distúrbios ambientais ou até mesmo pelo tempo de realização entre os inventários ter sido insuficiente pra recrutamento de novos indivíduos. Peixoto et al. (2012) afirmaram que a recorrência de fogo em local queimado recentemente, pode causar menor mortalidade que um incêndio em local que há muito tempo não queima. Assim sendo, 
em áreas de Cerrado protegidas do fogo, as taxas de recrutamento populacional, podem ser afetadas positiva e negativamente, dependendo da intensidade e frequência destes eventos (Roitman et al., 2008).

Quando se analisa as populações que foram mais representativas nestas duas fitofisionomias (CSS e CD), observa-se que Magonia pubescens, Qualea grandiflora e Qualea parviflora foram as que se destacaram em termos de densidade. Sendo que Magonia pubescens se comportou de maneira diferente entre CSS e CD, tendo maior recrutamento e acréscimo de área basal no CSS e foi a população que mais perdeu indivíduos no CD, tendo baixo recrutamento e decréscimo em área basal. Porém, ainda assim, foi superior a muitas outras espécies nesta fitofisionomia (Tabela 1). Comparando o número de indivíduos, nos dois inventários, observou-se que as populações de maior densidade foram as que mais perderam indivíduos, corroborando com os resultados de Felfili (1995) e Shen et al. (2013), possivelmente porque essas populações possuem elevada quantidade de indivíduos.

Já na FES, boa parte das populações (7) a mortalidade foi superior ao recrutamento, sendo este superior a mortalidade nas populações de Protium heptaphyllum, Faramea hyacinthina, Protium spruceanum e Eugenia acutata. Pela contagem de Poisson, em quatro populações as mudanças foram significativas, e em sete não significativas. A população de Protium spruceanum foi a que mais perdeu indivíduos, porém, foi a que mais recrutou, e esse resultado refletiu na área basal que foi maior no segundo inventário.

Em nove populações $(81,8 \%)$ as mudanças na área basal foram positivas, ou seja, houve aumento da área basal ao longo do intervalo avaliado (Tabela 1). Já para Ocotea odorífera e Terminalia glabrescens $(18,2 \%)$ foram registrados decremento em biomassa (mudanças negativa) (Tabela 1). Notou-se que na FES, a ação do vento ocasionou pequenas mudanças na estrutura das populações. De acordo com Appollinário et al. (2005) e Silva, Araújo (2009), as Florestas Semideciduais Tropicais frequentemente apresentam flutuações, com eventuais desequilíbrios, entre às taxas de mortalidade e de recrutamento, bem como de perda e ganho de biomassa. E em muitos casos, tais mudanças estão associadas com a reconstrução do ciclo silvigenético das comunidades florestais, especialmente logo após distúrbios naturais (Appollinário et al., 2005; Higuchi et al., 2008; Machado \& Oliveira-Filho, 2010).

De maneira geral é esperado que as populações se comportassem de maneira diferente pós-distúrbios, com algumas respondendo de forma positiva (ganho de indivíduos e aumento da área basal), e outras de forma negativa (perda de indivíduos e redução da área basal), o que indicaria possíveis dificuldades de estabelecimento (Machado \& Oliveira-Filho, 2010).

Assim, no presente estudo as populações do Cerrado (CSS e CD), não apresentaram a mesma tendência nas categorias de desenvolvimento (mortalidade, recrutamento e crescimento diamétrico). Hoffmann (1999) afirma que os efeitos do fogo em nível populacional variam consideravelmente entre as espécies. Assim sendo, o padrão aqui observado pode ser considerado dentro do esperado para áreas de Cerrado.

Assim, se analisado as populações das áreas de Cerrado, verifica-se que apenas Bowdichia virgilioides e Caryocar brasiliense, classificadas como secundária tardia e sem caracterização (Apêndice 1), não perderam indivíduos, cresceram em área basal e apresentaram taxas de mudanças em número de indivíduos e de incremento em área basal positivas, sendo assim, consideradas como mais resilientes que as demais espécies secundárias iniciais, tardias e sem caracterização, consideradas no estudo (Tabela 1).

Já as populações de Leptolobium dasycarpum, Hyptidendron canum, classificadas como secundária inicial, e Roupala montana (secundária tardia), (Apêndice 1), obtiveram mudanças negativas em área basal. Este padrão pode ser explicado principalmente pela alta taxa de mortalidade e baixo recrutamento e incremento, demonstrando certa sensibilidade frente aos distúrbios registrados nos fragmentos. O que sugere que essas espécies pertencem a um grupo que necessitam de maior tempo para se recuperar pós-distúrbios. De fato, a espécie Roupala montana, em diferentes fitofisionomias do Cerrado, já foi citada entre as espécies mais sensíveis aos incêndios (Hoffmann \& Solbrig, 2003; Mews et al., 2013; Santos et al., 2017).

A ocorrência periódica dos incêndios pode colocar em risco a diversidade de espécies (Silva et al., 2011), visto que grande maioria das populações em CSS e CD tiveram decréscimo 
em abundância e área basal, sendo poucas as espécies com resposta positiva, o que diminui a equabilidade e riqueza da comunidade, consequentemente acarreta na perda de diversidade.

Em ambas as fitofisionomias do Cerrado (CSS e CD) houve predominância de populações de espécies classificadas como secundária inicial e de dispersão anemocórica, que é uma guilda comum em diversos estudos no Cerrado (Oliveira et al., 2014, 2018), pois a anemocoria é facilitada pela redução foliar durante a estação seca (Mantovani \& Martins,1988). Nestes ambientes as populações de espécies secundárias iniciais foram as que mais perderam indivíduos.

Na FES quanto aos grupos ecológicos houve predominância das espécies classificadas, em número de indivíduos, como secundárias tardia e inicial, respectivamente (Apêndice 1). Espécies do grupo das secundárias tardias apresentaram as maiores taxas de recrutamento e mortalidade e foram as que mais perderam indivíduos. Já quanto a síndrome de dispersão todas as populações selecionadas dessa fitofisionomia possuem dispersão zoocórica (Apêndice 1), indicando assim, a importância da contribuição da fauna para a dispersão e manutenção das populações, e corrobora com os resultados de Yamamoto et al. (2007). A predominância das espécies secundárias e zoocóricas nesse fragmento pode ser considerado forte indício de que esta fitofisionomia se encontra em estágio mais avançado de sucessão.

De maneira geral, o que se observou com o estudo foi que os padrões temporais (variações nas taxas de dinâmica) entre fitofisionomias estudadas, ainda não são totalmente claros. Assim sendo, é importante a continuidade dos inventários nos três fragmentos, para verificar de forma mais perceptível quais são os padrões das flutuações temporais das vegetações estudadas, e o quanto estas serão influenciadas em cenários futuros com ausência de incêndios ou com a permanência destes.

\section{CONCLUSÃO}

Nas três fitofisionomias ocorreram mudanças na estrutura populacional. Na maioria das populações, a mortalidade foi superior ao recrutamento resultando em, redução do número de indivíduos. Em CSS e CD, esta resposta pode estar relacionada ao fogo. Na FES, as mudanças nas populações foram menos acentuadas, a mortalidade e o recrutamento foram maiores em populações mais densas. A maioria das populações mesmo com recrutamento inferior a mortalidade ocorreu aumento de área basal.

\section{REFERÊNCIAS}

Alvares, C. A., Stape, J. L., Sentelhas, P. C., Moraes, G., Leonardo, J., \& Sparovek, G. (2013). Koppen's climate classification map for Brazil. Meteorologische Zeitschrift, 22(6), 711-728. http://dx.doi.org/10.1127/0941-2948/2013/0507.

Angiosperm Phylogeny Group - APG IV. (2016). An update of the Angiosperm Phylogeny Group classification for the orders and families of flowering plants: APG IV. Botanical Journal of the Linnean Society, 181(1), 1-20. http://dx.doi.org/10.1111/boj.12385.

Appollinário, V., Oliveira-Filho, A. T., \& Guilherme, F. A. G. (2005). Tree population and community dynamics in a Brazilian tropical semideciduous Forest. Revista Brasileira de Botânica, 28(2), 247-360. http://dx.doi.org/10.1590/S0100-84042005000200014.

Cubas, R., Watzlawick, L. F., \& Figueiredo Filho, A. (2016). Incremento, ingresso, mortalidade em um remanescente de floresta ombrófila mista em Três Barras - SC. Ciência Florestal, 26(3), 889-900.

Ehrlén, J., \& Morris, Y. B. (2015). Predicting changes in the distribution and abundance of species under environmental change. Ecology Letters, 18(3), 303-314. PMid:25611188. http://dx.doi.org/10.1111/ele.12410.

Enoki, T., Kusumoto, B., Igarashi, S., \& Tsuji, K. (2014). Stand structure and plant species occurrence in forest edge habitat along different aged roads on Okinawa Island, southwestern Japan. Journal of Forest Research, 19(1), 97-104. http://dx.doi.org/10.1007/s10310-012-0383-9.

Felfili, J. M. (1995). Growth, recruitment and mortality in the Gama gallery forest in central Brazil over a six-year period (1985-1991). Journal of Tropical Ecology, 11(1), 67-83. http://dx.doi.org/10.1017/S0266467400008415. 
Felfili, J. M., Rezende, A. V., Júnior, M. C. D. S., \& Silva, M. A. (2000). Changes in the floristic composition of cerrado sensu stricto in Brazil over a nine-year period. Journal of Tropical Ecology, Cambridge, 16(4), 579-590. http://dx.doi.org/10.1017/S0266467400001589.

Gandolfi, S., Leitão-Filho, H. F., \& Bezerra, C. L. F. (1995). Levantamento florístico e caráter sucessional das espécies arbustivo-arbóreas de uma floresta mesófila semidecídua no município de Guarulhos, SP. Revista Brasileira de Biologia, 55(4), 753-767.

Gomes, L., Maracahipes, L., Marimon, B. S., Reis, S. M., Elias, F., Maracahipes-Santos, L., Marimon-Junior, B. H., \& Lenza, E. (2014). Post-fire recovery of savanna vegetation from rocky outcrops. Flora, 209(34), 201-208. http://dx.doi.org/10.1016/j.flora.2014.02.006.

Higuchi, P., Oliveira-Filho, A. T., Silva, A. C., Machado, E. L. M., Santos, R. M., \& Pifano, D. S. (2008). Dinâmica da comunidade arbórea em um fragmento de Floresta Estacional Semidecidual montana em Lavras, Minas Gerais, em diferentes classes de solos. Revista Árvore, 32(3), 417-426. http://dx.doi.org/10.1590/S0100-67622008000300004.

Hoffmann, W. A. (1999). Fire and population dynamics of woody plants in a neotropical savanna: matrix model projections. Ecology, 80(4), 1354-1369. http://dx.doi.org/10.1890/00129658(1999)080[1354:FAPDOW]2.0.CO;2.

Hoffmann, W. A., \& Solbrig, O. T. (2003). The role of topkill in the differential response of savanna woody species to fire. Forest Ecology and Management, 180(1-3), 273-286. http://dx.doi.org/10.1016/S03781127(02)00566-2.

Korning, J., \& Balslev, H. (1994). Growth and mortality of trees in Amazonian tropical rain forest in Ecuador. Journal of Vegetation Science, 5(1), 77-86. http://dx.doi.org/10.2307/3235641.

Lenza, E., Abadia, A. C., Menegat, H., Lúcio, N. W., Maracahipes-Santos, L., Mews, H. A., Santos, J. O., \& Martins, J. (2017). Does fire determine distinct floristic composition of two Cerrado savanna communities on different substrates? Acta Botanica Brasílica, 31(2), 250-259. http://dx.doi.org/10.1590/0102-33062016abb0198.

Lima, E. S., Lima, H. S., \& Ratter, J. A. (2009). Mudanças pós-fogo na estrutura e composição da vegetação lenhosa em um cerrado mesotrófico no período de cinco anos (1997-2002) em Nova Xavantina - MT. Cerne, 15(4), 468-480.

Lopes, S. F., Schiavini, I., Prado Júnior, J. Á., Gusson, A. E., Souza Neto, A. R., Vale, V. S., \& Dias Neto, O. C. (2011). Caracterização ecológica e distribuição diamétrica da vegetação arbórea em um remanescente de floresta estacional na Fazenda Experimental do Gloria, Uberlândia, MG. Bioscience Journal, 27(2), 322-335.

Machado, E. L. M., \& Oliveira-Filho, A. T. (2010). Spatial patterns of tree community dynamics are detectable in a small (4 ha) and disturbed fragment of the Brazilian Atlantic forest. Acta Botanica Brasílica, 24(1), 250-261. http://dx.doi.org/10.1590/S0102-33062010000100027.

Mantovani, W., \& Martins, F. R. (1988). Variações fenológicas das espécies do cerrado da Reserva Biológica de Moji Guaçu, Estado de São Paulo. Revista Brasileira de Botânica, 11, 101-112.

Melo, A. C. G., \& Durigan, G. (2010). Impacto do fogo e dinâmica da regeneração da comunidade vegetal em borda de Floresta Estacional Semidecidual (Gália, SP, Brasil). Revista Brasileira de Botânica, 33(1), 37-50. http://dx.doi.org/10.1590/S0100-84042010000100005.

Mews, H. A., Marimon, B. S., Maracahipes, L., Franczak, D. D., \& Marimon-Junior, B. H. (2011). Dinâmica da comunidade lenhosa de um Cerrado Típico na região Nordeste do Estado de Mato Grosso, Brasil. Biota Neotropica, 11(1), 73-82. http://dx.doi.org/10.1590/S1676-06032011000100007.

Mews, H. A., Silverio, D. V., Lenza, E., \& Marimon, B. S. (2013). Influência de agrupamentos de bambu na dinâmica pós-fogo da vegetação lenhosa de um cerrado típico, Mato Grosso, Brasil. Rodriguésia, 64(2), 211-221. http://dx.doi.org/10.1590/S2175-78602013000200002.

Miguel, E. P., Rezende, A. V., Leal, F. A., Pereira, R. S., \& Melo, R. R. D. (2016). Floristic-structural characterization and successional group of tree species in the cerrado biome of Tocantins State, Brazil. Revista Caatinga, Mossoró, 29(2), 393-404. http://dx.doi.org/10.1590/198321252016v29n216rc.

Nunes, Y. R. F., Mendonça, A. V. R., Botezelli, L., Machado, E. L. M., \& Oliveira-Filho, A. T. (2003). Variações da fisionomia, diversidade e composição de guildas da comunidade arbórea em um fragmento de floresta semidecidual em Lavras, MG. Acta Botanica Brasílica, 17(2), 213-229. http://dx.doi.org/10.1590/S0102-33062003000200005.

Oliveira, A. K. M., Resende, U. M., \& Schleder, E. J. D. (2014). Espécies vegetais e suas síndromes de dispersão em um remanescente de cerrado (sentido restrito) do município de Campo Grande, Mato Grosso do Sul. Ambiência, Guarapuava (PR), 10(2), 565-580. 
Oliveira, L. M., Sousa, R. M., Correa, N. E. R., Santos, A. F., \& Giongo, M. (2018). Florística e síndromes de dispersão de um fragmento de cerrado ao sul do estado do Tocantins. Scientia Agraria Paranaensis, Marechal Cândido Rondon, 17(1), 104-111.

Oliveira-Filho, A. T. (2006). Catálogo das árvores nativas de Minas Gerais: mapeamento e inventário da flora nativa e dos reflorestamentos de Minas Gerais (1. ed., 423 p.). Lavras: Ed. UFLA.

Otoni, T. J. O., Pereira, I. M., Oliveira, M. L. R., Machado, E. L. M., Farnezi, M. M., \& Mota, S. L. L. (2013). Componente arbóreo, estrutura fitossociologica e relações ambientais em um remanescente de cerradão, em Curvelo - MG. Cerne, Lavras, 19(2), 201-211. http://dx.doi.org/10.1590/S010477602013000200004.

Paiva, L. V., Araújo, G. M., \& Pedron, F. (2007). Structure and dynamics of a woody plant community of a tropical semi-deciduous seasonal forest in the "Estação Ecológica do Panga", municipality of Uberlândia, Minas Gerais, Brazil. Revista Brasileira de Botânica, 30(3), 365-373. http://dx.doi.org/10.1590/S0100-84042007000300003.

Peixoto, K. S., Sanchez, M., Pedroni, F., Ribeiro, M. N., Facure, K. G., Gomes-Klein, V. L., \& Guilherme, F. A. G. (2012). Dinâmica da comunidade arbórea em Floresta Estacional Semidecidual sob queimadas recorrentes. Acta Botanica Brasílica, 26(3), 697-708. http://dx.doi.org/10.1590/S010233062012000300020.

Pereira, I. M., Botelho, A. S., Berg, E. V. D., Oliveira-Filho, A. T., \& Machado, E. L. M. (2010). Caracterização ecológica de espécies arbóreas ocorrentes em ambientes de mata ciliar, como subsidio à recomposição de áreas alteradas nas cabeceiras do rio grande, Minas Gerais, Brasil. Ciência Florestal, 20(2), 235-253. http://dx.doi.org/10.5902/198050981849.

Pivello, V. R., Oliveras, I., Miranda, H. S., Haridasan, M., Sato, M. N., \& Meirelles, S. T. (2010). Effect of fires on soil nutrient availability in an open savanna in Central Brazil. Plant and Soil, 337(1-2), 111-123. http://dx.doi.org/10.1007/s11104-010-0508-x.

Ribeiro, J. F., \& Walter, B. M. T. (2008). As principais fitofisionomias do bioma Cerrado. In S. M. Sano, S. P. Almeida \& J. F. Ribeiro (Eds.), Cerrado: ecologia e flora (pp. 151-212). Brasília, DF: Embrapa Cerrados/Embrapa Informação Tecnológica.

Rios, M. N. S., Sousa-Silva, J. C., \& Malaquias, J. V. (2018). Mudanças pós-fogo na florística e estrutura da vegetação arbóreo-arbustiva de um Cerrado Sentido Restrito em Planaltina - DF. Ciência Florestal, 28(2), 469-482. http://dx.doi.org/10.5902/1980509832028.

Roitman, I., Felfili, J. M., \& Rezende, A. V. (2008). Tree dynamics of a fire-protected cerrado sensu stricto surrounded by forest plantations, over a 13-years period (1991-2004) in Bahia, Brazil. Plant Ecology, 197(2), 255-267. http://dx.doi.org/10.1007/s11258-007-9375-9.

Santos, L. M., Santos, J. O., Reis, S. M., \& Lenza, E. (2017). Temporal changes in species composition, diversity, and woody vegetation structure of savannas in the Cerrado-Amazon transition zone. Acta Botanica Brasílica, 32(1), 1-10.

Sheil, D., Jennings, S., \& Savill, P. (2000). Long-term permanent plot observations of vegetation dynamics in Budongo, a Ugandan rain forest. Journal of Tropical Ecology, 16(6), 865-882. http://dx.doi.org/10.1017/S0266467400001723.

Sheil, D., \& May, R. M. (1996). Mortality and recruitment rate evaluations in heterogeneous tropical forests. Journal of Ecology, Oxford, 84(1), 91-100. http://dx.doi.org/10.2307/2261703.

Shen, Y., Santiago, L. S., Ma, L., Lin, G. J., Lian, J. Y., Cao, H. L., \& Ye, W. H. (2013). Forest dynamics of a subtropical monsoon forest in Dinghushan, China: recruitment, mortality and the pace of community change. Journal of Tropical Ecology, Cambridge, 29(2), 131-145. http://dx.doi.org/10.1017/S0266467413000059.

Silva, M. D., Loiola, P. P., Rosatti, N. B., Silva, I. A., Cianciaruso, M. V., \& Batalha, M. A. (2011). Os efeitos dos regimes de fogo sobre a vegetação de Cerrado no Parque Nacional das Emas, GO: considerações para a conservação da diversidade. Biodiversidade Brasileira, 1(2), 26-39.

Silva, M. R., \& Araújo, G. M. (2009). Dinâmica da comunidade arbórea de uma floresta semidecidual em Uberlândia, MG, Brasil. Acta Botanica Brasílica, 23(1), 49-56. http://dx.doi.org/10.1590/S010233062009000100006.

Silva, W. M., Zorzanelli, J. P. F., Moreau, J. S., Abreu, K. M. P., \& Kunz, S. H. (2017). Estrutura e sucessão ecológica de uma comunidade florestal urbana no sul do Espírito Santo. Rodriguésia, 68(2), 301-314. http://dx.doi.org/10.1590/2175-7860201768202.

Toniato, M. T. Z., \& Oliveira-Filho, A. T. (2004). Variations in tree community composition and structure in a fragment of tropical semideciduous forest in southeastern Brazil related to different human 
disturbance histories. Forest Ecology and Management, 198(1-3), 319-339. http://dx.doi.org/10.1016/j.foreco.2004.05.029.

Van der Pijl, L. (1982). Principles of dispersal in higher plants. Nova York: Springer-Verlag. http://dx.doi.org/10.1007/978-3-642-87925-8.

Veloso, H. P., Rangel-Filho, A. L. R., \& Lima, J. C. A. (1991). Classificação da vegetação brasileira adaptada a um sistema universal (123 p.). São Paulo: Fundação Instituto Brasileiro de Geografia e Estatística.

Yamamoto, L. F., Kinoshita, L. S., \& Martins, F. R. (2007). Síndromes de polinização e de dispersão em fragmentos da Floresta Estacional Semidecídua Montana, SP, Brasil. Acta Botanica Brasílica, 21(3), 553-573. http://dx.doi.org/10.1590/S0102-33062007000300005.

Zar, J. H. (1996). Biostatistical analysis. New Jersey: Prentice-Hall.

Contribuição dos autores: LSS: writing - original draft, writing - review \& editing, investigation, TJOO: writing review \& editing, formal analysis, ADV: writing - review \& editing, APDG: writing - review \& editing, IMP: writing review \& editing, ELMM: writing - review \& editing, investigation, formal analysis, SAPF: writing - review \& editing. 
APÊNDICE 1. CLASSIFICAÇÃO EM GRUPOS SUCESSIONAIS E SÍNDROMES DE DISPERSÃO DAS ESPÉCIES AMOSTRADAS EM FITOFISIONOMIAS DE CERRADO (CSS = CERRADO STRICTO SENSU, CD = CERRADÃO) E FLORESTA ESTACIONAL SEMIDECIDUAL (FES) NA FAZENDA DO MOURA, CURVELO, MG.

\begin{tabular}{|c|c|c|c|}
\hline \multirow{2}{*}{ Espécies } & \multirow{2}{*}{ Fitofisionomia } & \multicolumn{2}{|c|}{ Guildas } \\
\hline & & Reg. & Dis \\
\hline Annona crassiflora Mart. & CSS & $\mathrm{Pi}$ & Zoo \\
\hline Annona sylvatica A. St.-Hil. & FES & $\mathrm{Pi}$ & Zoo \\
\hline Aspidosperma tomentosum Mart & CSS & $\mathrm{Pi}$ & Ane \\
\hline Astronium fraxinifolium Schott & $\begin{array}{l}C D \\
C S S\end{array}$ & St & Ane \\
\hline Bowdichia virgilioides Kunth & CSS & St & Ane \\
\hline Byrsonima coccolobifolia Kunth & CSS & $\mathrm{Si}$ & Zoo \\
\hline Byrsonima verbascifolia (L.) DC. & CSS & $\mathrm{Pi}$ & Zoo \\
\hline Caryocar brasiliense Cambess. & CSS & Sc & Zoo \\
\hline Copaifera langsdorffii Desf. & FES & Sc & Zoo \\
\hline Coutarea hexandra (Jacq.) K. Schum. & FES & $\mathrm{Si}$ & Zoo \\
\hline Diospyros burchellii Hiern & CSS & $\mathrm{Si}$ & Zoo \\
\hline Erythroxylum deciduum A. St.-Hil. & CSS & $\mathrm{Si}$ & Zoo \\
\hline Erythroxylum suberosum A. St.-Hil. & CSS & $\mathrm{Si}$ & Zoo \\
\hline Eugenia acutata Miq. & FES & $\mathrm{Si}$ & Zoo \\
\hline Faramea hyacinthina Mart. & FES & $\mathrm{Si}$ & Zoo \\
\hline Hyptidendron canum (Pohl ex Benth.) Harley & $C D$ & $\mathrm{Si}$ & Zoo \\
\hline Kielmeyera coriacea Mart. \& Zucc. & CSS & $\mathrm{Si}$ & Ane \\
\hline Kielmeyera grandiflora (Wawra) Saddi & $C D$ & $\mathrm{Si}$ & Ane \\
\hline Lafoensia vandelliana Cham. \& Schltdl & CSS & St & Ane \\
\hline Leptolobium dasycarpum Vogel & CSS & $\mathrm{Si}$ & Ane \\
\hline Machaerium opacum Vogel & $\begin{array}{l}\text { CD } \\
\text { CSS }\end{array}$ & $\mathrm{Si}$ & Ane \\
\hline Magonia pubescens A. St.-Hil. & $\begin{array}{l}C D \\
\text { CSS }\end{array}$ & $\mathrm{Si}$ & Ane \\
\hline Maytenus gonoclada Mart. & FES & St & Zoo \\
\hline Ocotea odorifera Rohwer & FES & St & Zoo \\
\hline Ouratea hexasperma (A. St.-Hil.) Baill. & CSS & $\mathrm{Si}$ & Zoo \\
\hline Piptocarpha rotundifolia (Less.) Baker & CSS & $\mathrm{Si}$ & Ane \\
\hline Plathymenia reticulata Benth. & CSS & St & Ane \\
\hline Plenckia populnea Reissek. & CSS & $\mathrm{Si}$ & Ane \\
\hline Pouteria ramiflora (Mart.) Radlk. & CSS & St & Ane \\
\hline Protium heptaphyllum (Aubl.) Marchand & $\begin{array}{l}C D \\
F E S\end{array}$ & $\mathrm{Si}$ & Zoo \\
\hline Protium spruceanum (Benth.) Engl. & FES & $\mathrm{Si}$ & Zoo \\
\hline Qualea multiflora Mart. & CSS & $\mathrm{Si}$ & Ane \\
\hline Qualea grandiflora Mart. & $\begin{array}{l}\text { CD } \\
\text { CSS }\end{array}$ & $\mathrm{Si}$ & Ane \\
\hline Qualea parviflora Mart. & $\begin{array}{l}\text { CD } \\
\text { CSS }\end{array}$ & $\mathrm{Si}$ & Ane \\
\hline Roupala montana Aubl. & $C D$ & $\mathrm{Si}$ & Aut \\
\hline Tachigali vulgaris L.G.Silva \& H.C.Lima & $C D$ & $\mathrm{Pi}$ & Ane \\
\hline Tabebuia aurea (Silva Manso) & $\begin{array}{l}C D \\
\text { CSS }\end{array}$ & St & Ane \\
\hline Tachigali aurea Tul. & $\begin{array}{l}C D \\
\text { CSS }\end{array}$ & St & Ane \\
\hline Terminalia argentea Mart. & $\begin{array}{l}C D \\
\text { CSS }\end{array}$ & $\mathrm{Si}$ & Ane \\
\hline Terminalia glabrescens Mart. & FES & $\mathrm{Si}$ & Zoo \\
\hline Tocoyena formosa (Cham. \& Schltdl.) K. Schum. & CSS & $\mathrm{Pi}$ & Zoo \\
\hline Trichilia pallens C. DC. & FES & St & Zoo \\
\hline Vatairea macrocarpa (Benth.) Ducke & $\begin{array}{l}C D \\
\text { CSS }\end{array}$ & St & Ane \\
\hline Xylopia aromática (Lam.) Mart. & $C D$ & $\mathrm{Si}$ & Zoo \\
\hline
\end{tabular}

Guildas de regeneração: $\mathrm{Pi}$ = pioneiras, $\mathrm{Si}$ = secundária inicial, $\mathrm{St}$ = secundária tardia. Guildas de dispersão: Ane $=$ anemocórica, Aut $=$ autocórica, $Z$ Zoo = zoocórica e sem caracterização. 